$\xi=-\mathrm{B}$

\title{
Estimating the Parameters of the Exponential-Geometric distribution based on progressively type-II censored data
}

\author{
Aisha Fayomi*, Hamdah Al-Shammari \\ King Abdulaziz University, Faculty of Sciences, Department of Statistics, Jeddah 21589, Saudi Arabia \\ *Corresponding author E-mail: afayomi@kau.edu.sa
}

\begin{abstract}
This paper deals with the problem of parameters estimation of the Exponential-Geometric (EG) distribution based on progressive type-II censored data. It turns out that the maximum likelihood estimators for the distribution parameters have no closed forms, therefore the EM algorithm are alternatively used. The asymptotic variance of the MLEs of the targeted parameters under progressive type-II censoring is computed along with the asymptotic confidence intervals. Finally, a simple numerical example is given to illustrate the obtained results.
\end{abstract}

Keywords: EM algorithm ; Exponential-Geometric distribution ; Maximum Likelihood Estimator ; Progressive type-II Censoring

\section{Introduction}

With no doubt, the analysis of lifetime data has become a very important subject in different scientific disciplines. A numerous lifetime distributions have shown how they are so important to model perfectly practical situations. In the literature, there are a huge number of papers dealing with distributions that have been developed by compounding some other life distributions. This is actually necessary to fulfill the need of modeling so many complicated real problems. For example, Adamidis and Loukas 1998, (see reference, ,1] ), introduced a two-parameter exponentialgeometric distribution with decreasing failure rate by compounding an exponential with a geometric distributions. The new proposed distribution, in fact, has some desirable properties and nice physical interpretations. Another distribution that can be considered as a counterpart of the EG distribution is established by [2], and is called the complementary exponential-geometric (CEG) distribution.

In real life, especially in scientific experiments, it is desirable to study a censored data rather than a complete data set. Censoring is a common technique in survival analysis and is used effectively in various fields such as statistics, engineering and medical disciplines. It appears when exact lifetimes of entities or components are only partially known. There are different types of censoring that can be considered; type-I, type-II, progressively type-II and hybrid censoring schemes. The type-I and type-II censoring schemes are known to be the most extensively used. A short summary of censoring types is given here: In type-I censoring, a life test is implemented for a fixed-time period, while in type-II censoring, an experiment stops when a pre-specified number of units fail. It is noted that both type-I and type-II do not allow for removal of elements at points rather than termination point of an experiment. Therefore, the progressive type-II has been introduced to overcome the problem of units' removals. For comprehensive overview on this topic, reader might be referred to [3].
The parameter estimation is a challenging task especially in the case of compounded distributions. For this reason, computational methods are often sought, see for example, [3] and references therein for a comprehensive review of results in this direction. Inference procedures based on the maximum likelihood estimators have been considered when the underlying distribution is Gaussian, Gumpertz, bivariate normal, generalized logistic and the lifetime of a $k$-unit parallel system distributions, see for more details, [4], [5], [6], [7] and [8]. In some cases, MLEs cannot be obtained in a closed form; therefore, numerical methods can be employed. For example, [9], [10] studied parameter estimation under progressive type-II censored data using the Expectation-Maximization (EM) algorithm. It is an alternative procedure to compute MLEs in cases where the observed (complete) data could be incomplete. For more details on how to apply the EM algorithm, see [11], [12], [13], [14] and [15].

In this paper, we mainly study the properties of the EG distribution and estimate the unknown parameters based on progressive type-II censored data. A continuous random variable $X$ is said to have EG distribution with parameters $\beta$ and $p$ if its probability density function (pdf) is given by

$$
f(x ; \theta)=\frac{\beta(1-p) e^{-\beta x}}{\left(1-p e^{-\beta x}\right)^{2}} ; x>0, \beta>0,0<p<1,
$$

where $\theta=(\beta, p)$; such that $\beta$ and $p$ are scale and shape parameters, respectively. The corresponding cumulative distribution function (cdf) and the survival function of EG distribution; respectively, are given by

$$
\begin{aligned}
& F(x ; \theta)=\frac{1-e^{-\beta x}}{1-p e^{-\beta x}} ; x>0, \beta>0,0<p<1, \\
& s(x ; \theta)=\frac{e^{-\beta x}(1-p)}{1-p e^{-\beta x}} ; x>0, \beta>0,0<p<1,
\end{aligned}
$$


The rest of the paper is structured as follows: In Section 2, the maximum likelihood estimators of the EG distribution (MLEs) based on progressive type-II censored data will be derived, using the EM algorithm. In Section 3, the asymptotic variance of the MLE of the targeted parameter will be computed along with their asymptotic confidence intervals. In Section 4, a numerical example will be introduced to illustrate the obtained results under different censoring schemes. Finally, a conclusion will be stated in Section 5 .

\section{Parameters estimation}

In progressively type-II censored scheme, $\boldsymbol{n}$ units are placed on a test at time zero, when $\boldsymbol{m}<\boldsymbol{n}$ failures to be observed and the censoring scheme $\boldsymbol{R}=\boldsymbol{R}_{1}, \boldsymbol{R}_{2}, \ldots, \boldsymbol{R}_{\boldsymbol{m}}$ are fixed before the experiment with $\boldsymbol{R}_{\boldsymbol{i}} \geq \mathbf{0}$ and $\sum_{i=\mathbf{1}}^{\boldsymbol{m}} \boldsymbol{R}_{\boldsymbol{i}}=\boldsymbol{n}-\boldsymbol{m}$ are specified. At the first failure time $\boldsymbol{X}_{\mathbf{1}: m: \boldsymbol{n}}, \boldsymbol{R}_{\mathbf{1}}$ units, chosen at random, are removed from the $\boldsymbol{n}-\mathbf{1}$ remaining units. At the second failure time $\boldsymbol{X}_{\mathbf{2}: \mathbf{m}: \boldsymbol{n}}, \boldsymbol{R}_{\mathbf{2}}$ randomly chosen units from the remaining $\boldsymbol{n}-$ $\mathbf{2}-\boldsymbol{R}_{\mathbf{1}}$ units are removed. The experiment continues until the $\mathbf{m}^{\text {th }}$ failure time $\boldsymbol{X}_{\boldsymbol{m}: \boldsymbol{m}: \boldsymbol{n}}$. At this time, all remaining units are removed, and $\boldsymbol{R}_{\boldsymbol{m}}=\boldsymbol{n}-\boldsymbol{m}-\sum_{i=1}^{\boldsymbol{m}-\mathbf{1}} \boldsymbol{R}_{\boldsymbol{i}}$. The maximum likelihood function is given in the following subsection.

\subsection{Maximum likelihood estimation}

Suppose that the failure times of $n$ units on experiment have a pdf and cdf given by (1) and (2) respectively. Then the likelihood function of $X=\left(X_{1}, \ldots, X_{n}\right)$ under progressively type-II censored sample is given by

$$
\begin{aligned}
L(X \mid \theta) & =C \prod_{i=1}^{m} f\left(x_{i}\right)\left[1-F\left(x_{i}\right)\right]^{R_{i}} \\
& =C \prod_{i=1}^{m} \frac{\beta(1-p) e^{-\beta x_{i}}}{\left(1-p e^{-\beta x_{i}}\right)^{2}}\left[\frac{(1-p) e^{-\beta x_{i}}}{\left(1-p e^{-\beta x_{i}}\right)}\right]^{R_{i}},
\end{aligned}
$$

where $C=n\left(n-R_{1}-1\right) \cdots\left(n-\sum_{i=1}^{m-1} R_{i}-m+1\right)$. The loglikelihood function with ignoring constant can be written as follows.

$$
\begin{aligned}
\mathcal{L}((X \mid \boldsymbol{\theta}) & =m \ln \beta+\sum_{i=1}^{m}\left(1+R_{i}\right) \ln (1-p)-\beta \sum_{i=1}^{m} x_{i}\left(R_{i}+1\right) \\
& =-\sum_{i=1}^{m}\left(R_{i}+2\right) \ln \left(1-p e^{-\beta x_{i}}\right)
\end{aligned}
$$

Consequently, the Fisher's score functions is given by

$$
\begin{aligned}
\frac{\partial \log \mathcal{L}}{\partial \beta}= & \frac{m}{\beta}-\frac{p \sum_{i=1}^{m}\left(R_{i}+2\right) x_{i} e^{-\beta x_{i}}}{\left(1-p e^{-\beta x_{i}}\right)} \\
& -\sum_{i=1}^{m}\left(R_{i}+1\right) x_{i} . \\
\frac{\partial \log \mathcal{L}}{\partial p}= & -\frac{\sum_{i=1}^{m}\left(R_{i}+1\right)}{(1-p)}+\frac{\sum_{i=1}^{m}\left(R_{i}+2\right) e^{-\beta x_{i}}}{\left(1-p e^{-\beta x_{i}}\right)} .
\end{aligned}
$$

The maximum likelihood estimators, say $\hat{\beta}$ and $\hat{p}$, of $\beta$ and $p$ are the solutions of the equations (6) and (7). We note that they cannot be achieved in closed forms. Therefore, we use the EM algorithm to solve these equations.

\subsection{Expectation-Maximization algorithm}

The EM algorithm is used to compute the estimators based on the maximum likelihood in the presence of missing or incomplete data Since the progressive type-II censoring can be considered as an incomplete data set (see [8] and [9]), therefore, the EM algorithm is an alternative method to find the MLEs numerically. The EM algorithm consists of an E-step (Estimation step) followed by an M-step (Maximization step) defined as follows:

E-step: Compute $Q\left(\theta ; \theta^{t}\right)$ where $Q\left(\theta ; \theta^{t}\right)=E_{\theta^{t}}[\ell(\theta ; x) \mid Y]$.

M-step: Find $\theta^{t+1}$ in $\Theta$ such that $Q\left(\theta^{t+1} ; \theta^{t}\right) \geq Q\left(\theta ; \theta^{t}\right)$ for all $\theta \in \Theta$.

The E-step and the M-step are repeated interchangeably until the difference $L\left(\theta^{t+1}\right)-L\left(\theta^{t}\right)$ is less than $\delta$, where $\delta$ is a small quantity. Now, assume that $Y=\left(Y_{1}, \ldots, Y_{m}\right)$ in such $Y_{1}<\cdots<Y_{m}$ represents the progressive type-II censored (observed) data from a population with pdf and cdf that given in equations (1) and (2), respectively. Further, let $Z=\left(Z_{1}, Z_{2}, \ldots, Z_{m-n}\right)$ be the censored (unobserved) data, which can be considered as missing data. The combination vector of $Y$ and $Z$, say $W=(X, Z)$, is the complete data. Then, the maximum likelihood estimates of $\theta=(\beta, p)$ based on the distribution of $W$, ignoring the additive constant, is

$$
\begin{aligned}
L_{C}(W ; \theta)= & n \ln (\beta)+\sum_{i=1}^{m}\left(R_{i}+1\right) \ln (1-p) \\
& -\beta \sum_{i=1}^{m} y_{i}\left(R_{i}+1\right) \\
& -\sum_{i=1}^{m}\left(R_{i}+2\right) \ln \left(1-p e^{-\beta y_{i}}\right) \\
& -\beta \sum_{i=1}^{n-m} z_{i}\left(R_{i}+1\right) \\
& -\sum_{i=1}^{n-m}\left(R_{i}+2\right) \ln \left(1-p e^{-\beta z_{i}}\right) .
\end{aligned}
$$

The E-step of the EM algorithm emerges from $L_{c}(W ; \theta)$ by substituting any function of $z_{i}$ say $g\left(z_{i}\right)$ with $L_{s}(W ; \theta)=$ $E\left[g\left(z_{i}\right) \mid z_{i}>y_{m}\right]$. Therefore, the log-likelihood function becomes

$$
\begin{aligned}
L_{S}(W ; \theta)=n \ln (\beta) & +\sum_{i=1}^{m}\left(R_{i}+1\right) \ln (1-p) \\
& -\beta \sum_{i=1}^{m} y_{i}\left(R_{i}+1\right) \\
& -\sum_{i=1}^{m}\left(R_{i}+2\right) \ln \left(1-p e^{-\beta y_{i}}\right) \\
& -(n-m)\left[\beta A\left(y_{m}, \theta\right)+B\left(y_{m}, \theta\right)\right],
\end{aligned}
$$

Here, $A\left(y_{m}, \theta\right)$ and $B\left(y_{m}, \theta\right)$ are as follows

$$
\left.\begin{array}{c}
A\left(y_{m}, \theta\right)=E\left[\left(R_{i}+1\right) z_{i} \mid z_{i}>y_{m}\right] \\
\left.y_{m}, \theta\right)=E\left[\left(R_{i}+2\right) \log \left(1-p e^{-\beta z_{i}}\right) \mid z_{i}>y_{m}\right]
\end{array}\right\}
$$

To solve the above two equations in Equation (9), the following theorem should to be stated first:

Theorem 1 Given a random variable $Y=\left(Y_{1}, Y_{2}, \ldots, Y_{m}\right)$ distributed as EG distribution, $Y \sim E G(\beta, p)$, the conditional distribution of $Z_{i} ; i=1,2,3, \ldots, n-m$, can be written as

$$
\begin{aligned}
f_{Z \mid Y}\left(Z_{i} \mid Y_{m: n}=y_{m: n}\right) & = \begin{cases}\frac{f\left(Z_{i} \mid \theta\right)}{\left[1-F\left(Y_{m: n} \mid \theta\right)\right]} & ; z_{i}>y_{m: n} \\
0 & ; \text { otherwise }\end{cases} \\
& =\left\{\begin{array}{cc}
\beta D \frac{e^{-\beta z_{i}}}{\left(1-p e^{-\beta z_{i}}\right)^{2}} & ; z_{i}>y_{m: n} \\
0 & ; \text { otherwise }
\end{array}\right.
\end{aligned}
$$

Here $D$ is a function of $y$ and is given by 


$$
D=\frac{\left(1-p e^{-\beta y_{m}}\right)}{e^{-\beta y_{m}}}
$$

A proof of the theorem can be found in details in ref. [9]. Therefore, the expectations in (10) can be written as follows:

$$
\begin{aligned}
& A\left(y_{m}, \theta\right)=-\frac{(R+1) D}{p \beta}[\ln (1-p)] \\
& B\left(y_{m}, \theta\right)=-(R+2) \frac{1}{p e^{-\beta y_{m}}}\left[\ln \left(1-p e^{-\beta y_{m}}\right)+p e^{-\beta y_{m}}\right]
\end{aligned}
$$

In M-step of the EM algorithm which involves the maximization of equation (9) with respect to $\theta$.If at the $k t h$ stage the estimate of $\theta$ is $\hat{\theta}^{(t)}$ then $\hat{\theta}^{(t+1)}$ is computed by maximizing the following equation:

$$
\begin{aligned}
g(\theta)= & n \ln (\beta)+\sum_{i=1}^{m}\left(R_{i}+1\right) \ln (1-p)-\beta \sum_{i=1}^{m} y_{i}\left(R_{i}+1\right) \\
& -\sum_{i=1}^{m}\left(R_{i}+2\right) \ln \left(1-p e^{-\beta y_{i}}\right) \\
& -(n-m)\left[\beta A\left(y_{m}, \hat{\theta}^{(t)}\right)+B\left(y_{m}, \hat{\theta}^{(t)}\right)\right] .
\end{aligned}
$$

Differentiating (12) with respect to $\theta=(\beta, p)$ and setting the results equal to 0 , yields the two following equations:

$$
\begin{aligned}
\frac{\partial g}{\partial \beta}= & \frac{n}{\beta^{(t+1)}}-\frac{p \sum_{i=1}^{m}\left(R_{i}+2\right) y_{i} e^{-\beta^{(t+1)}} y_{i}}{\left(1-p e^{-\beta^{(t+1)}} y_{i}\right)} \\
& -\sum_{i=1}^{m}\left(R_{i}+1\right) y_{i}-(n-m) A\left(y_{m}, \hat{\theta}^{(t)}\right)=0 \\
\frac{\partial g}{\partial p}= & -\frac{\sum_{i=1}^{m}\left(R_{i}+1\right)}{\left(1-p^{(t+1)}\right)}+\frac{\sum_{i=1}^{m}\left(R_{i}+2\right) e^{-\beta y_{i}}}{\left(1-p^{(t+1)} e^{\left.-\beta y_{i}\right)}\right.}=0
\end{aligned}
$$

Next, we find $\hat{\theta}^{(t)}=\left(\widehat{\beta}^{(t+1)}, \hat{p}^{(t+1)}\right)$ by solving fixed-point type equations, $h(\theta)=\theta$, where

$$
h(\beta)=n\left[\begin{array}{c}
\frac{p \sum_{i=1}^{m}\left(R_{i}+2\right) y_{i} e^{-\beta^{(t+1)}} y_{i}}{\left(1-p e^{-\beta^{(t+1)} y_{i}}\right)} \\
+\sum_{i=1}^{m}\left(R_{i}+1\right) y_{i}+(n-m) A\left(y_{m}, \hat{\theta}^{(t)}\right)
\end{array}\right]^{-1}
$$

and

$$
h(p)=1-1-\sum_{i=1}^{m}\left(R_{i}+1\right)\left[\frac{\sum_{i=1}^{m}\left(R_{i}+2\right) e^{-\beta y_{i}}}{\left(1-p^{(t+1)} e^{-\beta y_{i}}\right)}\right]^{-1} .
$$

Subsequently, the E-step and the M-step are then repeated until convergence is achieved to the desired level of accuracy.

\section{Asymptotic variance}

In this section, we compute the asymptotic variance of MLEs using the idea of missing information principle see [9]. In addition, the asymptotic variance of MLEs can be used to construct the asymptotic confidence intervals through the observed information. The idea of missing information principle can be expressed through the following:

$$
I_{Y}(\theta)=I_{W}(\theta)-I_{Z \mid Y}(\theta),
$$

where $I_{Y}(\theta)$ is the observed information, $I_{W}(\theta)$ is the complete information and $I_{Z \mid Y}(\theta)$ is the (unobserved) missing information. The complete information matrix is
$I_{W}(\theta)=-E\left[\frac{\partial^{2} l_{c}(W, \theta)}{\partial \theta^{2}}\right]$,

where $l_{c}(W, \theta)$ is the log-likelihood function of the complete data. The missing information matrix can be written as

$I_{Z \mid Y}(\theta)=-(n-m) E\left[\frac{\partial^{2} \ln f_{Z \mid Y}(Z \mid y)}{\partial \theta^{2}}\right]$.

Now, the complete information matrix of $Y=\left(Y_{1}, \ldots, Y_{n}\right)$ where $Y$ is a progressive type-II censored data selected from a population with pdf and cdf given in equations (1) and (2), respectively, is given as follows:

$$
\begin{aligned}
I_{W}(\beta) & =-E\left[\frac{\partial^{2} l_{c}(W, \theta)}{\partial \beta^{2}}\right] \\
& =\frac{n}{\beta^{2}}-p \sum_{i=1}^{m}\left(R_{i}+2\right) E\left[\frac{y_{i}^{2} e^{-\beta y_{i}}}{\left(1-p e^{-\beta y_{i}}\right)^{2}}\right] . \\
I_{W}(p) & =-E\left[\frac{\partial^{2} l_{c}(W, \theta)}{\partial p^{2}}\right] \\
& =\frac{\sum_{i=1}^{m}\left(R_{i}+1\right)}{(1-p)^{2}}-\sum_{i=1}^{m}\left(R_{i}+2\right) E\left[\frac{e^{-2 \beta y_{i}}}{\left(1-p e^{-\beta y_{i}}\right)^{2}}\right] .
\end{aligned}
$$

The logarithm of the conditional distribution is given by

$$
\begin{aligned}
\ln f_{Z \mid Y}(Z \mid y)= & \ln (\beta)+\ln \left(1-p e^{-\beta y_{m}}\right)+\beta y_{m}-\beta Z \\
& -2 \ln \left(1-p e^{-\beta z}\right) .
\end{aligned}
$$

Next, the expectations of the second order derivative of (15) with respect to $\theta=(\beta, p)$ are:

$$
\begin{aligned}
E\left[\frac{\partial^{2} \ln f_{Z \mid Y}(Z \mid y)}{\partial \beta^{2}}\right]= & -\frac{1}{\beta^{2}}-\frac{p y_{m}{ }^{2} e^{-\beta y_{m}}}{\left(1-p e^{-\beta y_{m}}\right)^{2}} \\
& +E\left[\frac{2 p z^{2} e^{-\beta z}}{\left(1-p e^{-\beta z}\right)^{2}}\right] . \\
E\left[\frac{\partial^{2} \ln f_{Z \mid Y}(Z \mid y)}{\partial p^{2}}\right]= & \frac{-e^{-2 \beta y_{m}}}{\left(1-p e^{-\beta y_{m}}\right)^{2}}+E\left[\frac{2 e^{-2 \beta z}}{\left(1-p e^{-\beta z}\right)^{2}}\right] .
\end{aligned}
$$

Therefore, the matrix $I_{Z \mid Y}(\theta)$, where $\theta=(\beta, p)$, can be written as follows:

$$
\begin{aligned}
& I_{Z \mid Y}(\beta)=(n-m)\left\{\frac{1}{\beta^{2}}+\frac{p y_{m}{ }^{2} e^{-\beta y_{m}}}{\left(1-p e^{-\beta y_{m}}\right)^{2}}\right. \\
&\left.-2 E\left[\frac{p z^{2} e^{-\beta z}}{\left(1-p e^{-\beta z}\right)^{2}}\right]\right\} . \\
& I_{Z \mid Y}(p)=(n-m)\left\{\frac{e^{-2 \beta y_{m}}}{\left(1-p e^{\left.-\beta y_{m}\right)^{2}}\right.}-2 E\left[\frac{e^{-2 \beta z}}{\left(1-p e^{-\beta z}\right)^{2}}\right]\right\} .
\end{aligned}
$$

Subsequently, the asymptotic variance of $\hat{\theta}=(\widehat{\beta}, \hat{p})$ can be obtained by taking the inverse of the matrix $I_{Y}(\theta)$. Appling the normality properties of MLEs, we can use $100 \%(1-\gamma)$ approximate confidence intervals of parameters $\theta=(\beta, p)$ as follow.

$$
\widehat{\beta} \pm z \alpha / 2 \sqrt{\operatorname{var}(\widehat{\beta})} \quad, \quad \widehat{p} \pm z \alpha / 2 \sqrt{\operatorname{var}(\widehat{p})}
$$

where $\operatorname{var}(\widehat{\beta})$ and $\operatorname{var}(\widehat{p})$ are asymptotic variance of $\hat{\theta}=(\widehat{\beta}, \widehat{p})$ and $z \alpha / 2$ is percentile of standard normal distribution with righttail probability $\frac{\gamma}{2}$. 


\section{Result and Discussions}

To assess the mathematical derivations and the behavior of the estimated parameters, a simulation study has been conducted using $\mathrm{R}$ software. The program has been designed to obtain the MLEs, $\hat{\theta}=(\hat{\beta}, \hat{p})$, and other important measurements such as mean squared error (MSE), bias and a 95\% confidence interval with lower and upper limits (LL, UL) along with its length. Samples of different sizes, $n=10,20,30,40,50,60,70,80,90,100$, have been generated from EG distribution. The process has been run based on 2000 iterations by setting $\theta=(\beta=1, p=0.9)$. Also, different censoring schemes have been considered at different number of failures $m$ to each sample. The obtained results are shown in two tables.

Table 1: The length of confidence intervals of MLEs of EG distribution under progressive type-II censored data for $\beta=1$ and $p=0.9$ with different sample size and fixed number of failures observed.

\begin{tabular}{|c|c|c|c|c|c|}
\hline$m$ & $n$ & $\begin{array}{l}\text { Length } \\
\hat{\beta}, \hat{p}\end{array}$ & $m$ & $n$ & $\begin{array}{l}\text { Length } \\
\hat{\beta}, \hat{p}\end{array}$ \\
\hline \multirow[t]{4}{*}{15} & 20 & 0.81735 & 50 & 60 & 0.45000 \\
\hline & & 0.13793 & & & 0.05742 \\
\hline & 30 & 0.57272 & & 70 & 0.38249 \\
\hline & & 0.05430 & & & 0.03407 \\
\hline \multirow[t]{4}{*}{18} & 20 & 0.85069 & & 80 & 0.32516 \\
\hline & & 0.14561 & & & 0.02509 \\
\hline & 30 & 0.52005 & & 90 & 0.29762 \\
\hline & & 0.04203 & & & 0.02552 \\
\hline \multirow[t]{6}{*}{25} & 30 & 0.74598 & & 100 & 0.27042 \\
\hline & & 0.15101 & & & 0.02335 \\
\hline & 40 & 0.61806 & 60 & 70 & 0.43663 \\
\hline & & 0.09461 & & & 0.05479 \\
\hline & 50 & 0.50023 & & 80 & 0.40866 \\
\hline & & 0.07328 & & & 0.05294 \\
\hline \multirow[t]{4}{*}{30} & 40 & 0.60046 & & 90 & 0.32432 \\
\hline & & 0.09558 & & & 0.02839 \\
\hline & 50 & 0.54362 & & 100 & 0.24488 \\
\hline & & 0.07391 & & & 0.01897 \\
\hline \multirow[t]{4}{*}{40} & 50 & 0.53822 & 70 & 80 & 0.40483 \\
\hline & & 0.07848 & & & 0.05364 \\
\hline & 60 & 0.47204 & & 90 & 0.30732 \\
\hline & & 0.05489 & & & 0.02423 \\
\hline
\end{tabular}

Table 1 shows the length of the $95 \%$ confidence intervals of the MLEs for the parameters of EG distribution under progressive type-II censored sample at starting point $\theta=(\beta=1, p=0.9)$ using different sample size and fixed number of failures observed. It has been noted that, for fixed number of failures and as the sample size increased, the lengths of the $95 \%$ confidence intervals tend to be narrower.

Table 2 presents the simulated values of MLEs, MSE, bias and (LL, UL) of $95 \%$ confidence interval along with its length, under the consideration of the censoring schemes for different samples sizes $n=10,20,30, \ldots, 100$ and different numbers of failures $m$ for parameters $\theta=(\beta=1, p=0.9)$. From this table we could conclude that regardless of the censoring schemes and at which point the censored units are removed from the sample, for increasing sample size; the estimated value of the parameters converges to the true value, and the MSE of the MLEs decreases. It is also noted that for $n=100$, complete case, as $m$ increases (i.e. from 50 to 100) the MSE values decrease too. However, this may not satisfy, in some cases, as the simulated samples have been generated randomly. Additionally, different censoring schemes have been used for different samples sizes.

\section{Conclusion}

The problem of estimating the unknown parameters of the EG distribution under progressive type-II censored was discussed. The EM algorithm was used to find the maximum likelihood estimators of the scale and shape parameters of the model. Furthermore, the asymptotic variance was derived along with the asymptotic confidence intervals. The simulation study was conducted using different censoring schemes and different samples sizes to figure out the MLEs numerically. In addition, the MSE, Bias and their confidence intervals were presented under various aspects. The results show that as the sample size increased the estimated values of the unknown parameters converge to the true values.

\section{Acknowledgement}

The authors thank the reviewers for their useful comments and suggestions on an earlier version of this article which led to this improved one. This article is a part of the Master Thesis of the second named author whose work was supervised by the first authors.

\section{References}

[1] K. Adamidis, S. Loukas, A lifetime distribution with decreasing failure rate, Statistics \& Probability Letters, 39 (1998) 35-42.

[2] F. Louzada, M. Roman, V.G. Cancho, The complementary exponential geometric distribution: Model, properties, and a comparison with its counterpart, Computational Statistics \& Data Analysis, 55 (2011) 2516-2524

[3] N. Balakrishnan, R. Aggarwala, Progressive censoring: theory, methods, and applications, Springer Science \& Business Media, Place Published, 2000.

[4] N. Balakrishnan, N. Kannan, C.-T. Lin, H.K.T. Ng, Point and interval estimation for Gaussian distribution, based on progressively TypeII censored samples, IEEE Transactions on Reliability, 52 (2003) 90-95.

[5] S. CHANG, T.-R. TSAI, Point and interval estimations for the Gompertz distribution under progressive type-II censoring, Metron, 61 (2003) 403-418.

[6] N. Balakrishnan, J.-A. Kim, Point and interval estimation for bivariate normal distribution based on progressively Type-II censored data, Communications in Statistics-Theory and Methods, 34 (2005) 1297-1347.

[7] A. Asgharzadeh, Point and interval estimation for a generalized logistic distribution under progressive type II censoring, Communications in Statistics - Theory and Methods, 35 (2006) 1685-1702.

[8] B. Pradhan, Point and interval estimation for the lifetime distribution of a k-unit parallel system based on progressively type-II censored data, Economic Quality Control, 22 (2007) 175-186.

[9] H. Ng, P. Chan, N. Balakrishnan, Estimation of parameters from progressively censored data using EM algorithm, Computational Statistics \& Data Analysis, 39 (2002) 371-386.

[10] B.M. Al-Zahrani, M.S. Gindwan, Estimating the parameter of the Lindley distribution under progressive type-II censored data, Electronic Journal of Applied Statistical Analysis, 8 (2015) 100-111.

[11] G. McLachlan, T. Krishnan, The EM algorithm and extensions, John Wiley \& Sons, Place Published, 2007.

[12] O. Cappé, Online EM algorithm for hidden Markov models, Journal of Computational and Graphical Statistics, 20 (2011) 728-749.

[13] V. Melnykov, I. Melnykov, Initializing the EM algorithm in Gaussian mixture models with an unknown number of components, Computational Statistics \& Data Analysis, 56 (2012) 1381-1395.

[14] L. Kang, R. Carter, K. Darcy, J. Kauderer, S.-Y. Liao, A fast Monte Carlo EM algorithm for estimation in latent class model analysis with an application to assess diagnostic accuracy for cervical neoplasia in women with AGC, Journal of applied statistics, 40 (2013) 2699.

[15] C. Xu, P.D. Baines, J.-L. Wang, Standard error estimation using the EM algorithm for the joint modeling of survival and longitudinal data, Biostatistics, 15 (2014) 731-744. 
Table 2: The MLES, MSE, Bias, and 95\% Confidence limits and Length of the MLEs of the EG distribution based on progressive Type-II censored data for $\beta=1$ and $\mathrm{p}=0.9$ with different sample size and different number of failures observed. for $\beta=1$ and $\mathrm{p}=0.9$

\begin{tabular}{|c|c|c|c|c|c|c|c|}
\hline$n$ & $m$ & Censoring Scheme & $\begin{array}{c}\text { MLE } \\
\hat{\theta}=(\hat{\beta}, \hat{p})\end{array}$ & MSE & Bias & (LCI, UCI) & Length \\
\hline \multirow[t]{6}{*}{10} & 5 & $(0,0,3,1,1)$ & 1.66388 & 0.82656 & 0.66388 & $(1.06736,2.26040)$ & 1.19303 \\
\hline & & & 0.90400 & 0.00138 & 0.00400 & $(0.83533,0.97267)$ & 0.13734 \\
\hline & 7 & $(3,0,0,0,0,0,0)$ & 1.09252 & 0.20420 & 0.09252 & $(0.51108,1.67396)$ & 1.16289 \\
\hline & & & 0.89496 & 0.00145 & -0.00504 & $(0.83066,0.95926)$ & 0.12861 \\
\hline & 9 & $(0 * 8,1)$ & 0.90648 & 0.15708 & -0.09352 & $(0.33203,1.48093)$ & 1.14890 \\
\hline & & & 0.89648 & 0.00385 & -0.00352 & $(0.78181,1.01115)$ & 0.22935 \\
\hline \multirow[t]{6}{*}{20} & 10 & $(0 * 6,4,3,2,1)$ & 1.36896 & 0.26403 & 0.36896 & $(0.95547,1.78245)$ & 0.82697 \\
\hline & & & 0.89996 & 0.00078 & -0.00004 & $(0.84849,0.95143)$ & 0.10295 \\
\hline & 15 & $(0 * 13,4,1)$ & 0.98160 & 0.13509 & 0.13509 & $(0.57293,1.39027)$ & 0.81735 \\
\hline & & & 0.89336 & 0.00160 & -0.00664 & $(0.82439,0.96233)$ & 0.13793 \\
\hline & 18 & $(0 * 17,2)$ & 0.98554 & 0.08401 & -0.01446 & $(0.56020,1.41088)$ & 0.85069 \\
\hline & & & 0.89926 & 0.00171 & -0.00074 & $(0.82645,0.97207)$ & 0.14561 \\
\hline \multirow[t]{6}{*}{30} & 15 & $(0 * 12,5,5,5)$ & 1.34608 & 0.23543 & 0.34608 & $(1.05972,1.63244)$ & 0.57272 \\
\hline & & & 0.89810 & 0.00034 & -0.00190 & $(0.87095,0.92525)$ & 0.05430 \\
\hline & 18 & $(0 * 17,12)$ & 1.10724 & 0.05195 & 0.10724 & $(0.84722,1.36726)$ & 0.52005 \\
\hline & & & 0.89552 & 0.00028 & -0.00448 & $(0.87450,0.91654)$ & 0.04203 \\
\hline & 25 & $(0 * 25,5)$ & 1.08082 & 0.05171 & 0.08082 & $(0.70783,1.45381)$ & 0.74598 \\
\hline & & & 0.91292 & 0.00168 & 0.01292 & $(0.83742,0.98842)$ & 0.15101 \\
\hline \multirow[t]{6}{*}{40} & 20 & $(0 * 16,5,5,5,5)$ & 1.56552 & 0.43857 & 0.56552 & $(1.32194,1.80910)$ & 0.48716 \\
\hline & & & 0.90570 & 0.00025 & 0.00570 & $(0.88271,0.92869)$ & 0.04598 \\
\hline & 25 & $(0 * 25,5,5,3,2)$ & 1.46574 & 0.26964 & 0.46574 & $(1.15671,1.77477)$ & 0.61806 \\
\hline & & & 0.92386 & 0.00116 & 0.02386 & $(0.87656,0.97116)$ & 0.09461 \\
\hline & 30 & $(0 * 25,3,3,2,1,1)$ & 1.02084 & 0.06607 & 0.02084 & $(0.72061,1.32107)$ & 0.60046 \\
\hline & & & 0.90240 & 0.00067 & 0.00240 & $(0.85461,0.95019)$ & 0.09558 \\
\hline \multirow[t]{6}{*}{50} & 25 & $(0 * 25,10,10,3,2)$ & 1.81910 & 0.71397 & 0.81910 & $(1.56899,2.06921)$ & 0.50023 \\
\hline & & & 0.92960 & 0.00124 & 0.02960 & $(0.89296,0.96624)$ & 0.07328 \\
\hline & 30 & $(0 * 26,15,3,1,1)$ & 1.23950 & 0.09143 & 0.23950 & $(0.96769,1.51131)$ & 0.54362 \\
\hline & & & 0.90228 & 0.00039 & 0.00228 & $(0.86533,0.93923)$ & 0.07391 \\
\hline & 40 & $(0 * 36,3,3,2,2)$ & 1.02142 & 0.02803 & 0.02142 & $(0.75231,1.29053)$ & 0.53822 \\
\hline & & & 0.90344 & 0.00046 & 0.00344 & $(0.86420,0.94268)$ & 0.07848 \\
\hline \multirow[t]{6}{*}{60} & 30 & $(0 * 24,5,5,5,5,5,5)$ & 1.42616 & 0.22214 & 0.42616 & $(1.22564,1.62668)$ & 0.40104 \\
\hline & & & 0.90298 & 0.00014 & 0.00298 & $(0.88369,0.92227)$ & 0.03858 \\
\hline & 40 & $(0 * 35,5,5,4,3,3)$ & 1.15124 & 0.06306 & 0.15124 & $(0.91522,1.38726)$ & 0.47204 \\
\hline & & & 0.90144 & 0.00024 & 0.00144 & $(0.87400,0.92888)$ & 0.05489 \\
\hline & 50 & $(0 * 47,5,0,5)$ & 0.91110 & 0.02962 & -0.08890 & $(0.68610,1.13610)$ & 0.45000 \\
\hline & & & 0.89788 & 0.00023 & -0.00212 & $(0.86917,0.92659)$ & 0.05742 \\
\hline \multirow[t]{6}{*}{70} & 45 & $(0 * 40,5,5,5,5,5)$ & 1.19830 & 0.05652 & 0.19830 & $(0.99273,1.40387)$ & 0.41115 \\
\hline & & & 0.90298 & 0.00031 & 0.00298 & $(0.88225,0.92371)$ & 0.04147 \\
\hline & 50 & $(0 * 48,10,10)$ & 1.02394 & 0.01300 & 0.02394 & $(0.83270,1.21518)$ & 0.38249 \\
\hline & & & 0.89950 & 0.00009 & -0.00050 & $(0.88247,0.91653)$ & 0.03407 \\
\hline & 60 & $(0 * 58,5,5)$ & 1.00164 & 0.04089 & 0.00164 & $(0.78332,1.21996)$ & 0.43663 \\
\hline & & & 0.90282 & 0.00035 & 0.00282 & $(0.87543,0.93021)$ & 0.05479 \\
\hline \multirow[t]{6}{*}{80} & 50 & $(0 * 49,10,10,10)$ & 1.47022 & 0.23056 & 0.47022 & $(1.30764,1.63280)$ & 0.32516 \\
\hline & & & 0.91946 & 0.00042 & 0.01946 & $(0.90691,0.93201)$ & 0.02509 \\
\hline & 60 & $(0 * 56,6,5,6,3)$ & 1.02088 & 0.03108 & 0.02088 & $(0.81655,1.22521)$ & 0.40866 \\
\hline & & & 0.89970 & 0.00024 & -0.00030 & $(0.87323,0.92617)$ & 0.05294 \\
\hline & 70 & $(0 * 68,5,5)$ & 0.94950 & 0.01463 & -0.05050 & $(0.74709,1.15191)$ & 0.40483 \\
\hline & & & 0.90178 & 0.00021 & 0.00178 & $(0.87496,0.92860)$ & 0.05364 \\
\hline \multirow[t]{6}{*}{90} & 50 & $(0 * 46,10,10,10,10)$ & 1.30784 & 0.11901 & 0.30784 & $(1.15903,1.45665)$ & 0.29762 \\
\hline & & & 0.90276 & 0.00008 & 0.00276 & $(0.89000,0.91552)$ & 0.02552 \\
\hline & 60 & $(0 * 57,10,10,10)$ & 1.14750 & 0.07093 & 0.14750 & $(0.98534,1.30966)$ & 0.32432 \\
\hline & & & 0.90128 & 0.00019 & 0.00019 & $(0.88709,0.91547)$ & 0.02839 \\
\hline & 70 & $(0 * 69,20)$ & 0.97186 & 0.02031 & -0.02814 & $(0.81820,1.12552)$ & 0.30732 \\
\hline & & & 0.89860 & 0.00009 & -0.00140 & $(0.88648,0.91072)$ & 0.02423 \\
\hline \multirow[t]{6}{*}{100} & 50 & $(0 * 45,10,10,10,10,10)$ & 1.29598 & 0.11145 & 0.29598 & $(1.16077,1.43119)$ & 0.27042 \\
\hline & & & 0.89860 & 0.00007 & -0.00140 & $(0.88693,0.91027)$ & 0.02335 \\
\hline & 60 & $(0 * 58,20,20)$ & 1.21526 & 0.05549 & 0.21526 & $(1.09282,1.33770)$ & 0.24488 \\
\hline & & & 0.90118 & 0.00004 & 0.00118 & $(0.89169,0.91067)$ & 0.01897 \\
\hline & 100 & $(0 * 100)$ & 1.00134 & 0.01556 & 0.00134 & $(0.80413,1.19855)$ & 0.39442 \\
\hline & & & 0.90028 & 0.03078 & 0.00028 & $(0.55950,1.24106)$ & 0.68157 \\
\hline
\end{tabular}

